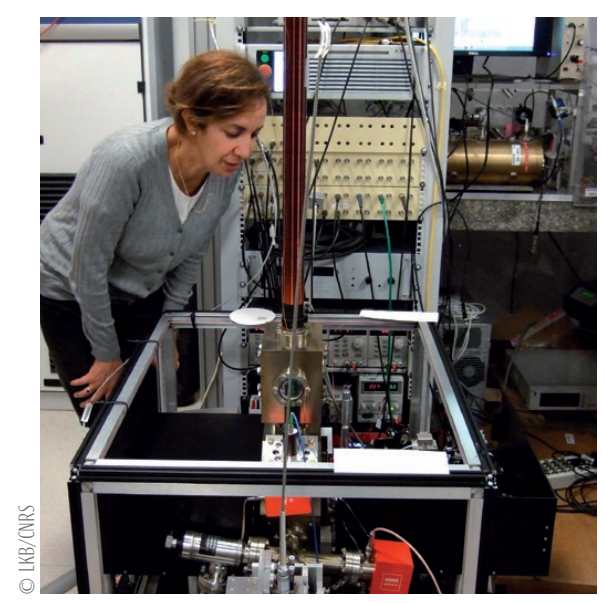

\title{
Saìda Guellati-Khélifa : à la recherche de la lumière pure
}

Professeur des universités et coresponsable du groupe Métrologie quantique et constantes fondamentales au Laboratoire Kastler Brossel (LKB), Saïda Guellati-Khélifa a coordonné avec I'Institut de Physique du CNRS la Nuit de la lumière au Musée des arts et métiers.

Pour Saïda Guellati-Khélifa, la lumière laser est plus qu'un outil : " un laser bien contrôlé permet de faire des expériences très propres et d'accéder à une sorte de pureté dans le geste expérimental ", dit-elle. Une pureté qui sied aux mesures physiques ultimes, grâce auxquelles les constantes physiques fondamentales sont déterminées et les tests des théories physiques poussés au bout. Dans la bouche de cette spécialiste de l'interaction lumière-matière, un autre mot revient plusieurs fois : rigueur. Si bien qu'à travers ses yeux, on en viendrait presque à considérer la physique comme une ascèse. Mais pas seulement. Car pour Saïda Guellati-Khélifa, c'est également une passion qu'elle partage avec le plus large public possible.

Chez cette scientifique d'origine algérienne, c'est une passion ancienne. "Étudiante, je me souviens avoir lu des articles de vulgarisation sur les travaux de Claude Cohen-Tannoudji sur le refroidissement d'atomes par laser, se remémore-t-elle. Ça m'a tout de suite séduit. " Mais c'est encouragée par un de ses professeurs qu'elle opte en 1988 pour le DEA Atomes, molécules et lasers à l'Université d'Orsay, après l'obtention d'une bourse d'études à l'étranger. "Tout ce que j'y ai appris me passionnait."

À la suite, Saïda Guellati-Khélifa pénètre l'univers rigoureux de la métrologie en préparant une thèse sur les horloges atomiques au césium. Dans un premier temps, elle applique les techniques de ralentissement par laser qui préparent les atomes à des mesures les plus précises possibles. Puis elle participe au développement de la première fontaine atomique, qu'elle utilisera pour mesurer avec une grande précision la fréquence de l'horloge à césium. "J'aime cette exigence propre à la métrologie qui fait que si tout n'est pas parfaitement sous contrôle, ça ne marche tout simplement pas ", explique l'expérimentatrice.

Recrutée comme maître de conférences au Conservatoire national des arts et métiers (CNAM) en 1993, Saïda Guellati-Khélifa évalue le potentiel du rubidium pour la conception d'horloges toujours plus précises. Les résultats sont décevants, mais le piège magnéto-optique qu'elle met alors au point lui permet de rejoindre l'équipe de François Biraben au LKB, cinq ans plus tard. Cette équipe utilise en effet des atomes de rubidium pour mesurer la constante de structure fine qui caractérise l'intensité des interactions entre la lumière et la matière.

Pendant plus de 10 ans, l'équipe qu'elle codirige désormais place tout son savoir-faire dans la mise au point de l'expérience la plus précise possible. Laser à la stabilité ultime, atomes refroidis au plus près du zéro absolu, capteur de vitesse précis au nanomètre par seconde près... Comme le résume la physicienne, "chaque décimale supplémentaire nécessite de pousser toujours plus loin chaque segment de l'expérience ". Avec, à la clé, en 2011, une mesure de la constante de structure fine avec une incertitude relative record de $7 \times 10^{-10}$ ! De quoi tester les prédictions de la théorie de l'électrodynamique quantique à un niveau jamais atteint auparavant.

Si Saida Guellati-Khélifa parle avec intensité des arcanes de la métrologie, elle pointe aussi le caractère parfois ingrat d'une discipline où le moindre détail abandonné au hasard ne pardonne pas. D'où l'importance pour elle d'échanger avec les autres, spécialistes ou grand public. "Cela offre le recul nécessaire à la remise en cause de ses propres certitudes ", analyse-t-elle.

En témoigne, par exemple, son implication auprès de la Société Française d'Optique, avec laquelle la spécialiste a participé à la mise au point d'une base de données destinée aux enseignants. De même, dans le cadre de l'Année internationale de la lumière, Saïda Guellati-Khélifa a représenté le CNAM au Comité national d'optique et de photonique et a participé à l'organisation de la Nuit de la lumière au Musée des arts et métiers, le 16 mai 2015. Des expériences y étaient présentées au grand public par les chercheurs. "Il s'agissait de montrer que certains aspects de la lumière sont au coeur des problématiques actuelles de recherche, à travers des expériences accessibles, explique la physicienne. Et ce, en brouillant le moins possible les notions de physique sous-jacentes, ce qui pour moi est essentiel. "Question de pureté, sans doute!

Mathieu Grousson, journaliste 